\title{
Identification of Putative Vero Cell Protein(s) that Bind Specifically to Recombinant Envelope Protein of Dengue Virus Type 2
}

\author{
Seema Zargar ${ }^{1 *}$, Tanveer A Wani ${ }^{2}$, SK Jain ${ }^{3}$ \\ ${ }^{1}$ Department of Biochemistry, King Saud University, PO Box 22452, ${ }^{2}$ Department of Pharmaceutical Chemistry, College of \\ Pharmacy, King Saud University, Riyadh, 11451, Kingdom of Saudi Arabia, ${ }^{3}$ Department of Biotechnology, Faculty of Science, \\ Jamia Hamdard, New Delhi, India
}

*For correspondence: Email: szargar@ksu.edu.sa; Tel: 966-11-8051692

\begin{abstract}
Purpose: To identify protein targets in host (vero) cell since there is currently no therapy or a licensed tetravalent vaccine to combat all the four virus serotypes of dengue virus.

Methods: The domain III of the dengue virus encoded envelope protein was expressed in pET28a expression vector and the purified recombinant protein was labeled with biotin without altering its immunogenicity. Vero cell proteins on nitrocellulose membrane reacted with recombinant envelope protein domain III to identify viral target proteins in vero cells.

Results: The $45 \mathrm{KDa}, 43 \mathrm{KDa}$ and $30 \mathrm{KDa}$ plasma membrane proteins were identified as viral envelope targets. Competitive binding assay showed these proteins competing with dengue virus binding. MTT assay indicate that viability of vero cells increases in cultures pretreated with $45 \mathrm{KDa}, 43 \mathrm{KDa}$ and 30 $K D$ a proteins before dengue infection.

Conclusion: These results indicate the possible role of these proteins in viral binding to vero cells. The study provides a preliminary insight that would aid in determining the target epitopes against protein $E$ domain III of dengue virus and hence, formulation of a vaccine for preparing neutralizing antibodies.
\end{abstract}

Keywords: Dengue virus envelope, Biotinylation, Ni-NTA purification, Target epitopes, Plaque assay, Competitive blocking assay

\begin{abstract}
Tropical Journal of Pharmaceutical Research is indexed by Science Citation Index (SciSearch), Scopus, International Pharmaceutical Abstract, Chemical Abstracts, Embase, Index Copernicus, EBSCO, African Index Medicus, JournalSeek, Journal Citation Reports/Science Edition, Directory of Open Access Journals (DOAJ), African Journal Online, Bioline International, Open-J-Gate and Pharmacy Abstracts
\end{abstract}

\section{INTRODUCTION}

Dengue virus belongs to the family Flaviviridae and includes immunologically four different serotypes (DEN-1, 2, 3 and DEN-4) [1]. The virus is transmitted through Aedes mosquito [2] and causes infections that range from dengue fever (DF) to life-threatening dengue hemorrhagic fever (DHF) and dengue shock syndrome (DSS). Despite current efforts to control dengue, based primarily on vector control and case management, the burden of the disease remains considerable. Dengue virus is a single-stranded, positive sense RNA virus with an $11 \mathrm{~kb}$ nonfragmented genome surrounded by a lipid bilayer envelope. RNA codes for three structural proteins and seven non-structural proteins. The three structural proteins are $C$ (nucleocapsid), $M$ (membrane associated protein) and $\mathrm{E}$ (envelope protein) and the seven non-structural proteins are designated as NS1, NS2, NS3, NS4, NS5, NS6 and NS7.

To establish infection virus requires entry into cells, followed by release of nucleocapsid. This is achieved by the fusion of the viral membrane 
with a cellular membrane $[3,4]$. The initial binding of dengue virus to target cells is mediated by binding of the envelope protein to a specific and unidentified cell surface receptor(s) [5].

Viral interaction with its targets observed by electron microscopy has been already reported [6]. The structure of the ectodomain of DEN-2E protein [7] shows it to be similar to that of the $E$ protein of the Tick-Borne Encephalitis virus (another member of the Flaviviridae family) [8]. The $E$ protein is organized into distinct domains designated as I, II and III. Of these, domain III (300 - 400aa), which has a single disulfide bond, is of particular importance from the viewpoint of vaccine development as it contains multiple serotype specific conformation-dependent, neutralizing epitopes and the host cell receptor recognition site [9]. The aim of present study was to identify the potential target proteins in the host cell that are involved in binding to E-protein to cause the infection.

\section{EXPERIMENTAL}

Preparation of dengue type 2 virus RNA and $E$ gene domain III coding sequence

Vero cells were grown at $37{ }^{\circ} \mathrm{C}$ in a humidified incubator using $5 \% \mathrm{CO}_{2}$, RPMI 1640 medium (Gibico, Grand Island, NY) supplemented with 10 $\%$ fetal bovine serum (FBS), penicillin and streptomycin. Cells were infected $(\mathrm{MOI}=0.1-$ 0.2 PFU/cell) with dengue type 2 virus (isolated clinically). Four days after infection, total RNA was extracted using QIAmp viral RNA mini kit (Qiagen, Chatsworth, CA), according to manufacturer's instructions. Viral genomic RNA was used as a template to obtain the DEN-2 virus $E$ gene fragment (aa 295 - 400) DNA by one-step RT-PCR (Qiagen), with parameters as follows: $50{ }^{\circ} \mathrm{C}$ for $30 \mathrm{~min}, 95{ }^{\circ} \mathrm{C}$ for $15 \mathrm{~min}$, and 40 cycles of $94{ }^{\circ} \mathrm{C}$ for $15 \mathrm{~s}, 55^{\circ} \mathrm{C}$ for $30 \mathrm{~s}, 72^{\circ} \mathrm{C}$ for $1 \mathrm{~min}$, with a final extension at $72{ }^{\circ} \mathrm{C}$ for 2 min. The sense strand primer was

5'-

GATGGATCCAAAGGAATGTCATATTCTATG-3' (the italicised sequence is BamHIrestriction site),

the antisense strand primer was

5'-

GATCTCGAGTTGGCCGATTGAGCTTCCTTT3' (italic sequence is Xhol restriction site).

After PCR, a $318 \mathrm{bp}$ of the DEN-2 E gene domain III sequence was obtained, with flanking BamHI and Xhol restriction sites.
Construction of recombinant expression plasmid and purification of recombinant Eprotein

The PCR product was purified with a Qiaquick DNA purification kit (Qiagen) and digested with BamHI and Xhol, then ligated into the corresponding BamHI and Xhol restriction sites in the expression vector pET28a (Novagen, Darmstadt, Germany), generating a recombinant plasmid, pET28a-DEN2EDIII. The construct was identified by $\mathrm{T} 7$ sequencing primer and compared with data reported for NGC strain sequence (Genbank accession no. D00346). The culture was grown and lysed as previously described [9]. The lysate was purified by Ni-NTA affinity matrix (Qiagen) and the flow-through was collected. The column was washed with $50 \mathrm{ml}$ wash buffer (20 mM phosphate buffer $\mathrm{pH} 7.5 / 300$ $\mathrm{mM} \mathrm{NaCl} / 20 \mathrm{mM}$ imidazole) and eluted sequentially with 100, 250, and $500 \mathrm{mM}$ imidazole containing buffers. The emergence of proteins was monitored by Bradford method. The protein-containing fractions were analyzed by SDS-PAGE as described by Laemmeli in 1970 [10]. The relevant fractions were pooled and dialyzed against PBS.

\section{Biotinylation of purified recombinant $E$ protein}

Sequence analysis of recombinant dengue type 2 envelope domains III shows 17 lysine (K) residues and hence was labelled with biotin. EZLink® Sulfo-NHS-Biotinylation kit was procured from Pierce and rDEN2EDIII was biotinylated according to supplier's protocol with minor modifications. Excess of biotin reagent was removed by desalting columns and HABA (4'hydroxyazobenzene-2carboxylic acid) assay was done for measuring the level of biotin incorporation. Label incorporation was estimated by adding the biotinylated protein to mixture of HABA dye and avidin and ODs were compared and values calculated using HABA calculator on http://www.piercenet/haba.com.

\section{Western blotting}

Total protein was extracted from infected vero cells by RIPA buffer and transferred to nitrocellulose membrane which was later blocked with $5 \mathrm{X}$ skimmed milk for $2 \mathrm{~h}$ at room temperature. Diluted biotin antibody (BTN4 from Thermo Scientific, Pierce) (final concentration 0.1 - $5 \mu \mathrm{g} / \mathrm{ml}$ ) was added and incubated overnight on shaker in $4{ }^{\circ} \mathrm{C}$. Next day membrane was washed 5 - 6 times with PBST and incubated with streptavidin HRP solution (1:5,000 dilution) (Pierce) for $2 \mathrm{~h}$ at room temperature. The 
membrane was again washed 5-6 times with PBS-T and hybridized with substrate reagent (2 mg DAB in PBST $+20 \mu \mathrm{L}$ of $30 \% \mathrm{H}_{2} \mathrm{O}_{2}$ ). Color development was achieved by gentle shaking for $5 \mathrm{~min}$ at RT and bands showing hybridization signals were detected.

\section{Treatment of vero cells with electroeluted proteins before dengue-2 challenge}

Vero cell line was cultured in RPMI1640 medium containing $10 \%$ fetal bovine serum. The culture was set in $25 \mathrm{~cm}^{2}$ tissue culture flasks and incubated to achieve a level of $80 \%$ confluences. Cultures were washed once with PBS before infection. For infection, the cells were passaged in 6,24 or 96 well plates to a confluence of $80 \%$. Each group of cells were infected with 5,10 and $20 \mu \mathrm{g}$ of purified recombinant $\mathrm{E}$ - protein for $30 \mathrm{~min}$ at room temperature and kept for rocking at room temperature for $1 \mathrm{~h}$ to check the proper concentration of recombinant protein to be used.

\section{Methylthiazol tetrazolium assay on cells}

Recombinant electro eluted protein was further purified with dialysis in 0.2 X RIPA buffer and serial dilution of purified protein was mixed with dengue virus type 2, NGC strain. The mixture was added to Vero cells to assess the ability to protect cells from DEN-2 virus infection. MTT assay was done to check the viability of cells before and after treatments as previously described [11] to determine the ability of the purified electro eluted proteins to block DEN-2 binding to Vero cells.In this process, the cells were passaged at $5 \times 10^{5}$ cells per well in 6-well plates and incubated at $33{ }^{\circ} \mathrm{C}$ with $5 \% \mathrm{CO}_{2}$ for $36-48$ h. Serial dilution of the purified rDEN2EDIII or BSA (as negative control) in RPMI medium was added to $0.5 \mathrm{ml}$ of DEN-2 strain in amount of $80-100$ pfu/well. Protein concentration in each case ranged from 0 to 10 $\mu \mathrm{g} / \mathrm{well}$. The mixtures were added onto Vero cells in 6 - well plates and incubated at $33{ }^{\circ} \mathrm{C}$ with $5 \% \mathrm{CO}_{2}$ for $1 \mathrm{~h}$. After aspiration of the protein/virus mixtures, RPMI medium (containing $0.8 \%$ agarose) was added to the well and incubated at $37{ }^{\circ} \mathrm{C}$ with $5 \% \mathrm{CO}_{2}$ for 3 days. 200 $\mu \mathrm{L}$ MTT (Methylthiazol Tetrazolium Assay) (5 $\mathrm{mg} / \mathrm{mL}$ in PBS) was added to the wells to stain the cells and the plaque numbers were scored.

\section{Statistical analysis}

Comparison between different groups was done using Independent samples t-test. $P \leq 0.05$ was considered statistically significant. All statistical calculations were done using Statistical Package for Social Science (SPSS) program, version (11.0).

\section{RESULTS}

\section{Cloning and expression of rDEN2EIII}

The domain III of dengue virus type 2 E-protein (318 bp) was obtained by reverse transcription PCR (Figure 1a). The amplified fragment was set up for digestion with BamH1 and Xho1. Vector pET 28a was also digested with the same enzymes and purified by gel extraction. The ligation was done to obtain the construct, pET28a-DEN2EIII. Colony PCR was done (Figure $1 \mathrm{~b}$ ) and the clone that gave a band at 318 bp was chosen for plasmid extraction. The recombinant plasmid was sequenced with T7 sequencing primer. Sequencing verified the insert that assembled to reference sequence from the NGC strain with $100 \%$ homology.

The recombinant plasmid supported the high level synthesis of domain III of E-protein and was further purified by Ni-NTA affinity chromatography under denaturing conditions. The purified protein gave a band at 16KDa on SDS-PAGE (Figure 2). Approximately $80-90 \%$ of the induced protein bound to the column, as evident from the comparison of a polypeptide profile of the lysate loaded on affinity matrix column with that of flow through material (Lane 2 ). Based on a comparison of the host band intensities in these two lanes, it appears that no or very little non-specific binding to the affinity matrix occurred. Elution with a buffer $(\mathrm{pH} 4.5)$ resulted in the emergence of highly purified recombinant protein from the column (lane1). Hence $>90 \%$ purity has been achieved for rD2EIII protein after expression in pET28a and purification on Ni-NTA affinity matrix. The yield of purified rD2EIII protein was $25-30 \mathrm{mg} / \mathrm{L}$ from induced culture.

\section{Biotinylation of purified recombinant protein rDEN2EIII}

Spectrophotometric reading of HABA-avidin (H/a) and that of HABA-avidin-biotinylated protein $(\mathrm{Ha} / \mathrm{b})$ showed incorporation of 1.61 moles of biotin/mole of protein. These calculations were made on the basis of HABA calculator available on pierce website. 


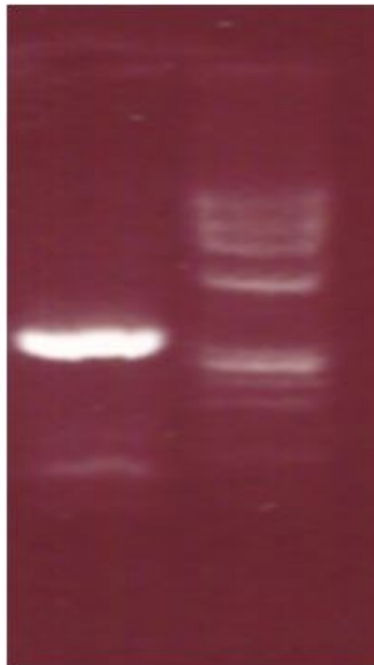

1 (a)

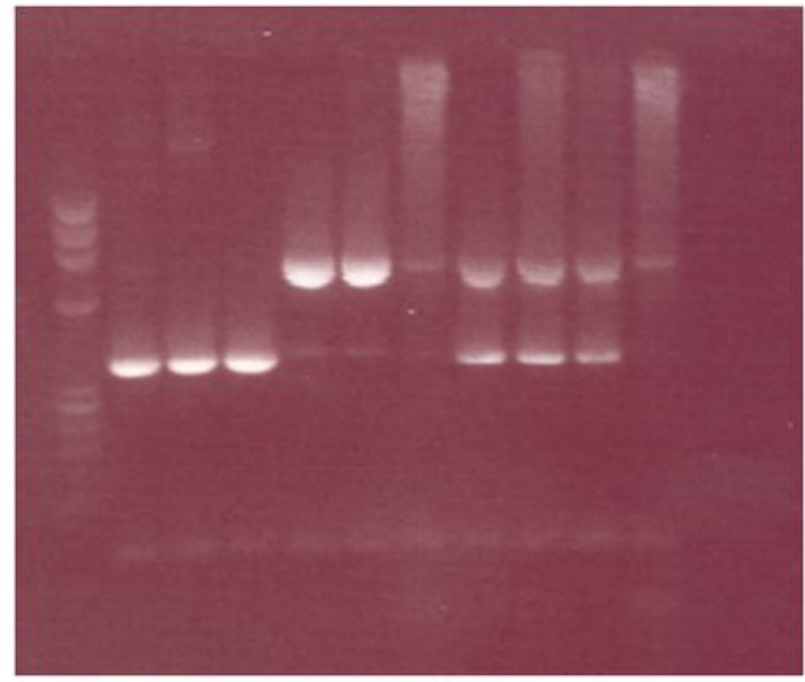

1 (b)

Figure 1: (a) PCR fragment of a $318 \mathrm{bp}$ of the DEN-2 E gene domain III sequence with flanking BamHI and Xhol restriction sites. Lane 1 is Haelll digest of $\Phi$ X174 from NEB and lane 2 is 318 bp fragments; (b) Colony PCR from several clones. The 318 bp band was obtained with same primers as used for PCR

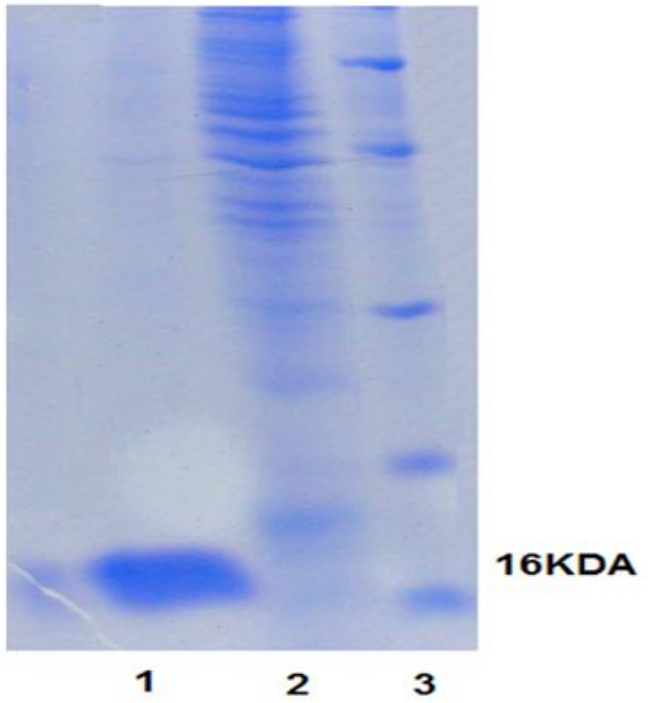

Figure 2: SDS-PAGE showing the purified DEN2E domain after Ni-NTA purification. Lane 1 (DEN2E), Lane 2 (flow through) showing high affinity of DEN2E to column and Lane 3 (marker)

\section{Identifying proteins that react to rDEN2EIII}

The total cellular protein from Vero cell was extracted with quantitative yield of nearly 1.3 $\mu \mathrm{g} / 10^{6}$ cells. When these gels were transferred to nitrocellulose membrane and hybridized with biotinylated envelope protein, signals at $45 \mathrm{KDa}$, $43 \mathrm{KDa}$ and $30 \mathrm{kDa}$ were obtained. No spot was seen in control gel (without rD2EIII) which means that signals were not due to internal biotin residues hence confirming that the interactions were specific.

\section{Evaluation of electro eluted vero cell proteins in vero cells by competitive blocking assay}

It was expected that the purified protein(s) have role in viral binding and hence they should alter dengue virus binding to these cells. When the cell cultures were pre-immune-stimulated with electro eluted proteins prior to dengue viral infections, the growth of cultures was more in comparison to the cultures that were not pre immunized or stimulated. It was found that the plaque forming units were less in pre-stimulated cell cultures of cells. There was significant $(p>$ $0.0001)$ difference between the groups prestimulated with 45 and $43 \mathrm{KDa}$ protein and normal group respectively, while the difference was non-significant $(p=0.873)$ between the group stimulated with $30 \mathrm{KDa}$ and normal group (Table 1).

\section{MTT assay}

Cell viability checked with MTT assay showed that all the three proteins increased the cell viability and decreased dengue virus interaction. The cells without pre-treatment with the above mentioned proteins had decreased cell viability and increased apoptosis (Figure 3). All the three purified proteins were added to vero cells before infection with equal quantity of $25 \mu \mathrm{M}$. It was found that $45 \mathrm{KDa}$ protein increased cell viability by 50 -fold approximately, $30 \mathrm{KDa}$ protein increased cell viability by $45 \%$ and $43 \mathrm{KDa}$ protein increased cell viability by approximately $25 \%$. 
Table 1: Averaged data from three replicate plaque assays of DENV2 titres in cell culture supernatants (each duplicate) after stimulation with eluted proteins

\begin{tabular}{lcc}
\hline Group & PFU/mL at M.0.I $\leq 10$ & $P$-value \\
& \pm SEM & $>0.0001^{*}$ \\
\hline Vero+RPMI+DEN & $6.23 \times 10^{4} \pm 1.41$ & $>0.0001^{*}$ \\
Vero+45KDa+DEN & $2.13 \times 10^{3} \pm 0.82$ & 0.873 \\
Vero+43KDa+DEN & $4.10 \times 10^{3} \pm 0.73$ & $5.67 \times 10^{4} \pm 3.60$ \\
Vero+30KDA+DEN & 5.60 is standard \\
\hline PFU = Plaque forming units. P values are for student's $t$ of comparisons with PBS treated cells; SEM is & &
\end{tabular}

\section{DISCUSSION}

The infective domain of dengue virus envelope is responsible for virus attachment and entry to host cells and is considered as a promising subunit for its neutralizing epitopes and host cells receptor recognition site. It has been reported that the $E$ protein maybe an effective subunit vaccine candidate because of its capacity to bind with host cell surface receptors. Also it has the ability to elicit protective antibodies against virus infection [12]. Recombinant E protein has been expressed in different expression systems like $E$. coli and $P$. pastoris $[13,14]$.

In the present study $E$ protein was expressed in $E$. coli and labeled with biotin after purification. The recombinant fusion protein could inhibit plaque formations in the host cell caused by

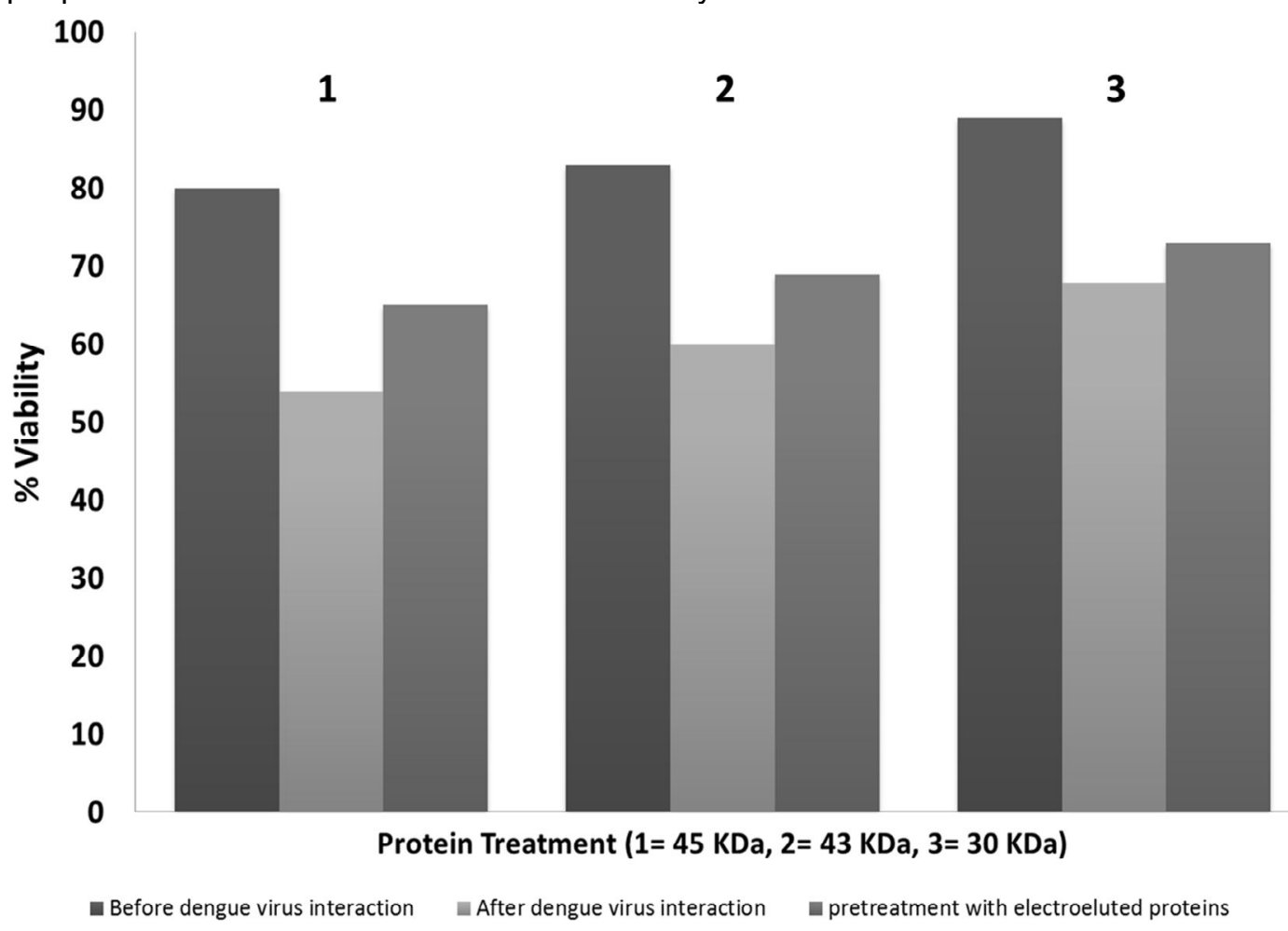

Figure 3: Effect of electro eluted proteins on cell viability. Cells were pretreated with $25 \mu \mathrm{M}$ of each purified protein. 1. Pre-treatment with $45 \mathrm{KDa}$ proteins 2. Pre-treatment with $43 \mathrm{KDa}$ protein and 3. Pre-treatment with 30 KDa proteins. Data shown are triplicates of three consecutive experiments dengue viral infections up to greater than $80 \%$. Recombinant domain III protein fused with His tag was checked for immunogenicity and it was again more than $90 \%$ active immunologically. An earlier study showed that the recombinant domain III protein fused with Protein A was not immunogenic. The high binding affinity of Protein $A$ to human immunoglobulins makes it impossible to use such proteins as a vaccine candidate [15]. In this study, a domain III fragment $(295$ - 400 aa) of DEN-2 E protein expressed in $E$. coli with a His tag carrier protein was produced because his tag is very small and doesn't interfere with E-protein's immunologic activity. His tag offers the advantage that it is poorly immunogenic and need not be removed from the recombinant protein. 
The use of the 6XHis tag obviates the need of subjecting the recombinant domain III protein to protease treatment and the inherent risk of recombinant protein degradation [16]. To identify the human cellular proteins interacting with the envelope protein of dengue virus serotype 2 inside host cells protein-protein interaction has been focused in present study. Protein-protein interaction studies are recent ideas for studying interaction of viral particles with host cells [1720].

In order to determine whether the purified recombinant protein retains the ability to bind with host cells or not, a competitive blocking assay was carried out. The result showed that the purified recombinant protein protects host cells from DEN-2 virus infection. It is likely that the recombinant protein was correctly refolded without loss of its biological function.

After verification of biological function, the recombinant $E$ protein was labeled by biotin. Biotin has a small size and hence does not alter the structure and functions of purified protein when used as a probe. We found the three signals at molecular weights $45 \mathrm{KDa}, 43 \mathrm{KDa}$ and $30 \mathrm{KDa}$. Also, there was no signal on nitrocellulose when only biotin without the dengue virus envelope was hybridized. This showed that the interaction was not because of internal biotin or biotinylated proteins in the cell but entirely specific to recombinant E-protein. Electro eluted $45 \mathrm{KDa}, 43 \mathrm{KDa}$ and $30 \mathrm{KDa}$ proteins added to vero cells prior to virus infection inhibited virus binding and increased cell viability. This feature may be attributed to decreased apoptosis in the cells due to pre stimulation by eluted proteins. It was previously described by us that infection with dengue virus increases apoptosis and decreases cell viability [6].

The ability of many viruses to manipulate the host antiviral immune response often results in complex host-pathogen interactions. DENVinfected cells are less able to produce antimicrobial peptides in response to secondary bacterial challenge.

\section{CONCLUSION}

The recombinant envelope protein produced is capable of inducing neutralizing antibodies which is consistent with previous studies. Furthermore, $45 \mathrm{KDa}, 43 \mathrm{KDa}$ and $30 \mathrm{KDa}$ proteins in vero cells that interact with dengue virus envelope protein have been identified. All these proteins are active immunologically.

\section{ACKNOWLEDGEMENT}

The authors would like to extend their sincere appreciation to the Deanship of Scientific Research at King Saud University for funding the Research Group no. RG-1435-073.

\section{REFERENCES}

1. Rosen $L M$, Drouet $T$, Deubel $V$. Detection of dengue virus RNA by reverse transcription-polymerase chain reaction in the liver and lymphoid organs but not in the brain in fatal human infection. Am J Trop Med Hyg 1999; 61(5):720-724.

2. Gubler DJ, Clark GG. Dengue/dengue hemorrhagic fever: the emergence of a global health problem. Emerg Infect Dis 1995; 1(2): 55-57.

3. Seema, Jain SK. Molecular mechanism of pathogenesis of dengue virus and its entry into target cell. Ind $\mathrm{J}$ of Clin Biochem 2005; 20(2): 92-103.

4. Hernandez LD, Hoffman LR, Wolfsberg TG, White JM. Virus-cell and cell-cell fusion. Annu Rev Cell Dev Biol 1996; 12(1): 627-661.

5. Feighny $R$, Burrous J, Mccown J, Hoke C, Putnak R. Purification of native dengue 2 viral protein and the ability of purified proteins to protect mice. Am J Trop Med Hyg 1992; 47(4): 405-412.

6. Zargar S, Wani TA, Jain SK. Morphological changes in vero cells postinfection with dengue virus type-2. Microsc Res Tech 2011; 74(4): 314-319.

7. Guo $X$, Yao $X$, Guowu B, Andrew DP, Yan $X$, Zhiyong $X$. Response of the mosquito protein interaction network to dengue infection. BMC Genomics 2010; 11(1): 380-387.

8. Megret MW, Hugnot JP, Falconar A, Gentry MK, Morens $D M$, Murray JM, Schlesinger JJ, Wright PJ, Young $P$, Regenmortel VMH. Use of recombinant fusion proteins and monoclonal antibodies to define linear and discontinuous antigenic sites on the dengue virus envelope glycoprotein. Virology 1992; 187(2): 480491.

9. Jaiswal $S$, Khanna $N$, Swaminathan $S$. High-level expression and one-step purification of recombinant dengue virus type 2 envelope domain III protein in Escherichia coli. Prot Expr Purif 2007; 33(2): 80-91.

10. Laemelli UK. Cleavage of structural proteins during the assembly of the head of bacteriophage T4. Nature 1970; 227:680-685.

11. Loosdrecht VAA, Beelen RH, Ossenkoppele GJ, Broekhoven MG, Langenhuijsen MM. A tetrazoliumbased colorimetric MTT assay to quantitate human monocyte mediated cytotoxicity against leukemic cells from cell lines and patients with acute myeloid leukemia. J Immunol Methods 1994; 174(1): 311-320.

12. Simmons $M$, Porter $K R$, Escamilla J, Graham R, Watts $D M$, Eckels $\mathrm{KH}$, Hayes CG. Evaluation of recombinant dengue viral envelope $B$ domain protein antigens for the detection of dengue complex-specific

Trop J Pharm Res, June 2015; 14(6): 1002 
antibodies. Am J Trop Med Hyg 1998; 58(2): $144-$ 151.

13. Sugrue RJ, Fu J, Howe J, Chan YC. Expression of the dengue virus structural proteins in Pichia pastoris leads to the generation of virus-like particles. Gen Virol 1997; 78(8): 1861-1866.

14. Chiu MW, Yang YL. Blocking the dengue virus 2 infections on BHK-21 cells with purified recombinant dengue virus $2 E$ protein expressed in Escherichia coli. Biochem Biophys Res Commun 2003; 309(16): 672-678

15. Sim S, Dimopoulos G. Dengue Virus Inhibits Immune Responses in Aedes aegypti Cells. PLoS ONE 2010; 5(5): e10678. doi:10.1371/journal.pone.0010678

16. Lee CW, Jung K, Jadhao SA, Suarez DL. Evaluation of chicken-origin (DF-1) and quail-origin (QT-6) fibroblast cell lines for replication of avian influenza viruses. J Virol Meth 2008; 153(1): 22-28.

17. Beatch MD, Everitt JC, Law LJ, Hobman TC. Interactions between rubella virus capsid an host protein p32 are important for virus replication. J Virol 2005; 79(16): 10807-10820.

18. Gao L, Aizaki H, He JW, Lai MM. Interactions between viral nonstructural proteins and host protein $h V A P-33$ mediate the formation of hepatitis $C$ virus RNA replication complex on lipid raft. J Virol 2004; 78(7): 3480-3488.

19. Staropoli I, Clement JM, Frenkiel MP, Hofnung M, Deubel V. Dengue virus envelope glycoprotein can be secreted from insect cells as a fusion with the maltose-binding protein. J Virol Meth 1996; 56(2): 179-189.

20. Hermida L, Rodriguez $R$, Lazo L, Silva $R$, Zulueta $A$, Chinea G, Lopez C, Guzman MG, Guillen G. A dengue-2 envelope fragment inserted within the structure of the P64k meningococcal protein carrier enables a functional immune response against the virus in mice. J Virol Meth 2004; 115(1): 41-49. 